\title{
DE3 潮汐的全球结构
}

陈泽宇, 吕达仁

中国科学院大气物理研究所中层大气和全球环境探测实验室, 北京 100029

E-mail: z.chen@mail.iap.ac.cn

2007-10-08 收稿, 2008-08-29 接受

中国科学院知识创新工程重要方向项目(批准号: KZCX2-YW-123, KGCX3-SYW-403)、自然科学基金重点项目(批准号: 40333034)、中国科学院 大气物理研究所自然科学创新项目(批准号: IAP070212)和武汉大学地球空间与大地测量教育部重点实验室项目(批准号: L036-02)资助

摘要 利用美国卫星 TIMED 在 2002 2006 年期间取得的温度数据(L2Aver1.07 SABER/TIMED 数据)提取了中间层和低热层(MLT)大气热力潮汐信号, 并且利用 Hough 模分解(HMD)方法考 察了 DE3 潮汐的全球结构. HMD 分析结果显示 DE3 潮汐主要受前两个 Hough 模的影响, 而包 括衰减模在内的其他 Hough 模的影响是可以忽略的. 根据 HMD 分析结果, 给出了 MLT 区域 中 DE3 潮汐活动的中心高度, 指出其活动的中心主要受第一传播 Hough 模, $(-3,3)$ 模的控制, 出现在 $110 \mathrm{~km}$ 高度, 表现出稳定的单峰年变化(出现在北半球夏季). 还发现 $(-3,3)$ 模表现出以 $2 \mathrm{a}$ 为周期的年际变化, 它同时清楚地反映在 $110 \mathrm{~km}$ 高度附近 DE3 潮汐振幅活动中. 在分析时 段内, 与 $(-3,3)$ 模 $2 \mathrm{a}$ 变化位相一致, DE3 潮汐在 2002 和 2004 年分别出现显著增强的活动, 其 中心振幅达到 $12 \mathrm{~K}$ 以上. 分析结果还指出, 在 $100 \mathrm{~km}$ 以下高度, 第二传播 Hough 模, $(-3,4)$ 模, 也起着重要的影响, 使得这里的 DE3 潮汐活动表现出关于赤道反对称的结构; 另外, $(-3,4)$ 模 表现出双峰年变化的特征, 并且在中间层下部区域起着显著的控制作用; 与此对应, 中间层下 部的 DE3 潮汐活动中心出现在冬末春初和秋末冬初两个季节中, 并且交替地出现在赤道以南 和赤道以北的热带地区.

关键词

DE3 潮汐

全球结构

两年变化

年变化

Hough 模分解
在中间层和低热层(MLT) 周日潮汐的各成分中, 向东传播并且纬向呈 3 波结构的潮汐是一个重要的 非迁移潮汐(DE3). 过去利用美国UARS卫星探测数 据得到的分析结果指出, 在 $95 \mathrm{~km}$ 高度这种潮汐是除 了迁移性周日潮汐外最显著的成分 ${ }^{[1 \sim 3]}$. Forbes和 $\mathrm{Wu}^{[4]}$ 通过利用 MLS/UARS卫星温度探测数据考察了 DE3 的结构, 结果显示在 $86 \mathrm{~km}$ 高度DE3 的振幅可以 达到 3 5 K, 虽然与该高度迁移性周日潮振幅 $8 \mathrm{~K}$ 相 比明显偏弱, 但是由于随季节变化的形式不同, DE3 达到振幅最大的时候, 其影响已经超过了迁移性周 日潮的影响. 近来, 陈泽宇和吕达仁 ${ }^{[5]}$ 利用为期 $1 \mathrm{a}$ 的 卫星温度数据开展分析的结果表明DE3 活动极大值 中心出现在更高的地方. 他们给出的结果显示在 97 $\mathrm{km}$ 高度DE3 振幅极大值可以达到 $9 \mathrm{~K}$. 与此同时, 近 来的关于电离层扰动的研究指出 DE3 潮汐可能对解
释一些特征扰动结构的形成有重要作用(请参考Wan 等人 ${ }^{[6]}$ 以及其中的文献). 在本研究工作中, 作者利用 多年卫星探测数据提取了高层大气潮汐信号, 同时 结合Hough模分解方法对 DE3 潮汐的全球结构及其 随时间的演变特征开展了进一步研究.

\section{1 数据和方法}

本研究工作使用了美国卫星探测数据, 即 $2 \mathrm{~A}$ 级 (L2A，1.06 版本)SABER/TIMED温度数据, 时间覆盖 从 2002 年 1 月到 2006 年 6 月. SABER是搭载在 TIMED卫星 ${ }^{[7]}$ 上的一个 10 通道宽带辐射计. 该设备 (以下简称SABER/TIMED) 在沿轨道飞行过程中采用 临边探测方案获取从对流层上部直到 $140 \mathrm{~km}$ 高度的 大气辐射, 在此基础上生成包括各种大气参量(温度、 气压和密度等)的廓线. 根据来自各验证报告的结果 
[8 11]指出在 10 100 km高度范围, SABER温度测量有 相当高的准确性, 误差平均为 $2 \sim 3 \mathrm{~K}$.

在本研究中采用了与陈泽宇和吕达仁 ${ }^{[5]}$ 中相同的 方案来分解潮汐信号. 这也是当前普遍使用的一种方 法 ${ }^{[12,13]}$, 其原理是利用TIMED卫星测量地方时(LST)存 在 12 min逐日进动的性质, 将 $60 \mathrm{~d}$ 测量数据归并在一 起构成一组在LST意义上连续覆盖一个整周日的数据 集(以下简称 $60 \mathrm{~d}$ 数据集); 在此基础上进一步将这些数 据归并到一组覆盖 $24 \mathrm{~h}$ 的时间段中. 这样，在某一空间 位置通过开展Fourier谐波拟合分析提取出各种周日谐 波信号. 以下将使用 $s$ 标记谐波的频率, $s=1,2,3$, 分别 对应 $24 \mathrm{~h}$ (周日), $12 \mathrm{~h}$ (半日)和 $8 \mathrm{~h}$ 周期的谐波.

为了得到单一的潮汐信号, 还需要进一步分解 空间波数成分. 首先在一组预设的标准空间坐标中 完成上述周日谐波分解; 在此基础上针对某种时间 谐波成分进一步利用那些覆盖同一个纬圈的该谐波 分析结果开展纬向波数谱分解过程, 这样就获得了 相应于该周日谐波的各潮汐成分. 以下将统一使用 $m$ 标记纬向波数, $m>0$ 和 $m<0$ 分别表示向西和向东传 播的潮汐成分. 结合上述对频率的标记规定, 每一种 潮汐成分可以用一个数对 $(s, m)$ 来标记. 于是标记 $\mathrm{DE} 3$ 潮汐的 $(s, m)$ 数对为 $(1,-3)$.

本文使用的空间坐标是根据 SABER/TIMED 空 间采样的方案建立的. 首先, 参考 SABER/TIMED 设 备在其切点处视场角的宽度, 在 20 140 km 高度范围 中按照 $2 \mathrm{~km}$ 间隔建立了标准垂直坐标. 另外, TIMED 卫星每日完成 15 次轨道飞行, 相邻轨道之间的经度 间隔为 $24^{\circ}, \mathrm{SABER} / \mathrm{TIMED}$ 在同一轨道中相临廓线 之间平均相距 3 纬度. 与这些空间采样方案对应, 水 平坐标中沿纬圈方向间隔为 $24^{\circ}$, 子午方向间隔为 $3^{\circ}$. 利用每一个 $60 \mathrm{~d}$ 数据集, 首先按照上述垂直坐标对 每一条测量廓线中的数据进行 $2 \mathrm{~km}$ 归并, 生成位于 标准垂直坐标上的数据廓线, 然后在上述空间坐标 中按照上述两步方案就完成了提取潮汐的工作.

在本研究中, 作者利用Hough模分解方法(以下 简称HMD方法) 对潮汐结构进行考察. 作为经典潮汐 理论的主要工具, Hough模代表了描述理想背景中大 气全球尺度波动方程(Laplace潮汐方程)的本征解. 而 这种经典线性理论 (Chapman和Lindzen ${ }^{[13]}$ ) 能够取得 成功的本质在于像潮汐这样的大气波动沿纬圈方向 的传播速度远远大于背景流动的速度, 这样可以忽 略由平流而带来的非线性效应. 因此, 在物理代表性
方面, Hough模分解的结果对解释潮汐信号的全球结 构给出了相当好的近似 ${ }^{[14]}$.

开展 HMD 分析需要待分解的潮汐信号在子午方 向具有充分覆盖. 然而, SABER 设备望远镜视线指向 卫星轨道的侧边, 其测量结果只能覆盖从一个半球 的 $50^{\circ}$ 纬度到另一半球 $85^{\circ}$ 纬度 (以下将 SABER 探测 达到 $85^{\circ}$ 的半球称为主半球). 因此, 在 $60 \mathrm{~d}$ 数据集中 满足充分周日数据采样的纬度范围(参见文献[5]中图 1), 不能满足开展 HMD 分析的要求. 为了提取充分 覆盖子午方向的潮汐信号, 作者根据 SABER/TIMED 每 $60 \mathrm{~d}$ 交换主半球的机动模式, 利用两个机动时段内 的数据, 即 $120 \mathrm{~d}$ 内的数据开展提取潮汐信号的工作, 这样得到的潮汐信号可以在子午方向覆盖南北半球 $80^{\circ}$ 之间的范围.

在本研究中使用了与DE3 潮汐对应的前 8 个 Hough模, 其中包括前 4 个传播模和前 4 个衰减模; 这些传播和衰减模分别包括两个相对于赤道对称和 两个反对称的模. 图 1 给出了这些作为纬度函数的 Hough模. 本文沿用了 Flattery ${ }^{[15]}$ 的标记方案, 用一对 整数 $(m, n)$ 来标记相应的Hough模. 这里, $m$ 是潮汐成 分的纬向波数, 对于 DE3 潮汐 $m=-3 ; n$ 是与这种潮汐 相关的的Hough模的指标. 具体针对DE3 潮汐而言, 前 4 个传播模分别对应 $n=3,4,5$ 和 6 ; 而前 4 个衰减 模分别对应 $n=-3,-4,-5$ 和-6. 另外, 由于本文只涉 及 DE3 潮汐, 为简化描述, 有时将省略波数指标 $m$, 并将 $(m, n)$ 模简记为 $H_{n}$.

另外需要指出, 作为纬度的函数, 图 1 中给出的 所有 Hough 模都已经沿子午方向进行了归一化, 如(1) 式所示.

$$
\int_{-1}^{1} H_{n}^{2}(\mu) \mathrm{d} \mu=1,
$$

这里, $\mu$ 是经过转换后的子午坐标, $\mu=\cos \theta$, $0^{\circ} \leqslant \theta \leqslant 180^{\circ} . \theta$ 为余纬, 原点位于 $90^{\circ} \mathrm{N}$.

$$
A \exp \left(\mathrm{i} \phi_{0}\right)=\sum_{n=-6,-5,-4,-3}^{3,4,5,6} C_{n} H_{n} 3 .
$$

(2)式概括地描述了开展 HMD 分析的过程. 在这 里, 为了简洁说明分解过程而采用了复数形式来表 达潮汐信号, 如(2)式左边所示, 其中 i 为虚数单位, $A$ 代表振幅, $\phi_{0}$ 代表潮汐初始相位; $A$ 和 $\phi_{0}$ 都是子午坐 标 $\mu$ 的函数. HMD 分析的原理就是将上述潮汐信号 用一组 Hough 模的线性组合来表达, 即(2)式右边的 


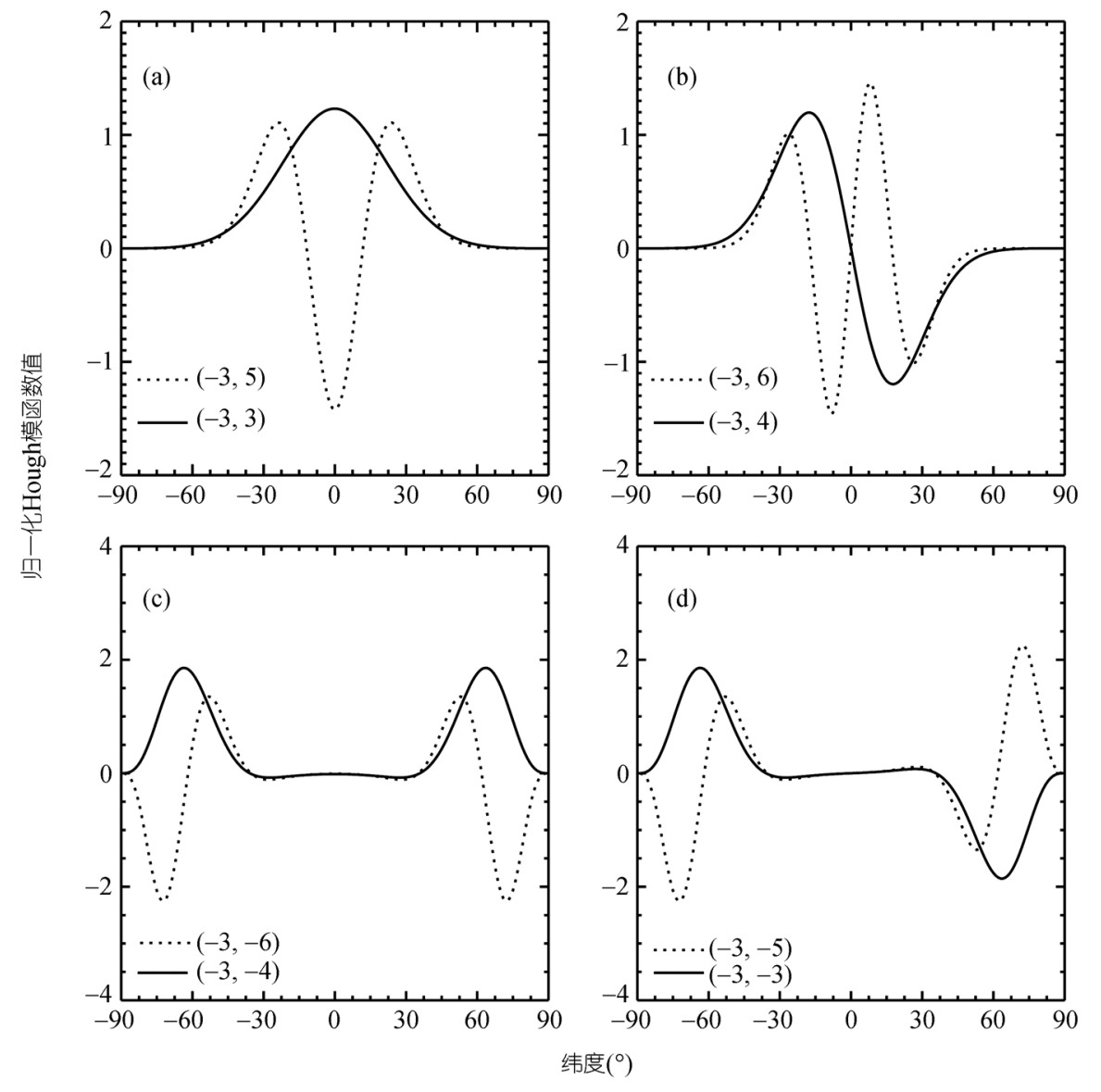

图 1 与 DE3 潮汐相关的头 8 个归一化 Hough 模

参见正文(1)式. 图例为 Hough 模标号

形式. 与等号左边的复数表达形式对应, 每一个 Hough 模的系数 $C_{n}$ 都应该是复数, 即 $C_{n}=C_{n, r}+\mathrm{i} C_{n, \mathrm{i}}$. HMD 分析过程的关键就是求出这些系数, 这样就可 以利用上述 Hough 模来重建潮汐.

另外, 由于这些 Hough 模都是沿子午方向归一 化的函数, 这为解释(2)中各 Hough 模系数 $C_{n}$ 的意义 带来了极大的方便. 利用(1)式, 将(2)式两边分别乘 以它们的共轭，然后沿子午方向积分后将得到(3)式, 其中上标*表示共轭复数. (3)式左边具有明显的意义, 它代表了沿子午方向所有纬圈中潮汐振幅平方的积 分, 具有能量的性质.

$$
\int_{-1}^{1} A^{2} \mathrm{~d} \mu=\sum_{n=-6,-5,-4,-3}^{3,4,5,6} C_{n} C_{n}^{*} .
$$

如果进一步将(3)式右边求和符号中的各项定义 为如(4)式所示的新参数 $N_{n}$, 不难看出这些新参数可 以简明地描述每一个 Hough 模对潮汐积分能量的贡
献; 同时也解释了潮汐的整体空间性质, 以下简称 $N_{n}$ 为 Hough 模振幅. 基于参数 $N_{n}$ 所具有的这些性质, 下面关于 DE3 潮汐全球结构的讨论主要是围绕这些 Hough 模的性质来进行.

$$
N_{n}^{2}=C_{n} C_{n}^{*}
$$

\section{2 分析结果}

根据(3)和(4)式, $N_{n}$ 描述了各 Hough 模对子午方 向潮汐积分能量的贡献, 这种贡献的大小同时说明 各 Hough 模的控制作用. 图 2 给出了 MLT 区域中各 高度上述 8 个 Hough 模振幅在分析时段(2002 2006 年)中的平均值, 它们隐含地描述了 DE3 潮汐的多年 平均结构. 图 2 显示 DE3 潮汐主要由头两个传播模组 成, 即 $(-3,3)$ 和 $(-3,4)$ 模; 其中 $(-3,3)$ 模起着主要的 控制作用; 除此而外, 包括 4 个衰减模在内的其他各 模态的贡献都是可以忽略的，这说明 MLT 区域中没 有显著的 DE3 潮汐源. 


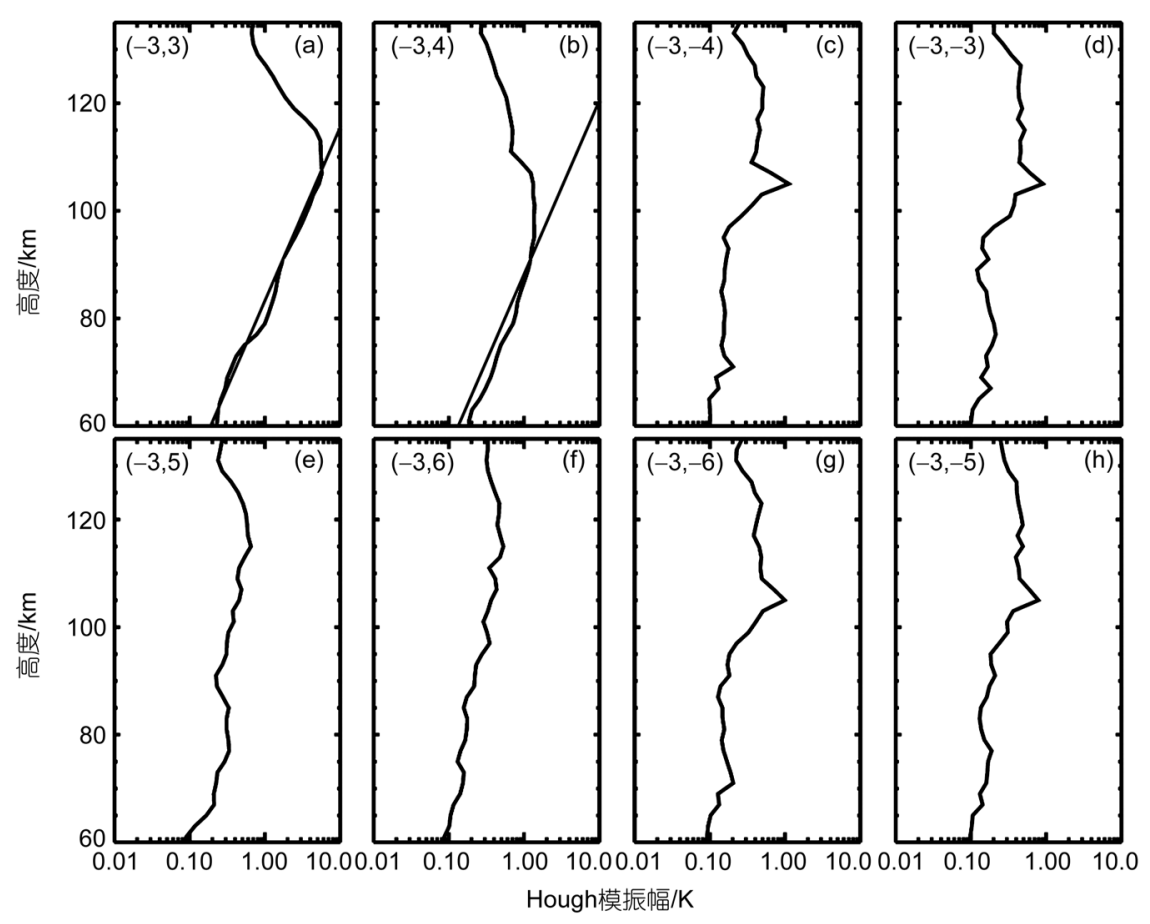

图 $22002 \sim 2006$ 年期间所有 8 个 Hough 模平均振幅 $N_{n}$ (参考(4)式)随高度的变化 其中, (a)和(b)中的参考直线分别指示与各自 Hough 模无耗散垂直传播状态对应的振幅随高度变化. 图例代表 Hough 模标号. 横坐标振幅(使用了对数坐标)

根据潮汐传播理论, 在无耗散背景中, 潮汐振幅 应该表现为随高度呈指数增长的形式, 即 $\propto \exp (z /$ $2 H), H$ 为大气标高. 为了考察上述两个Hough模的垂 直传播性质，图 2(a)和(b)中分别给出了一条描述这 种关系的参考直线(在MLT中 $H$ 约为 $7 \mathrm{~km}$, 注意横坐 标采用了对数刻度). 可以看到, 在 $90 \mathrm{~km}$ 高度以下, $(-3,3)$ 和 $(-3,4)$ 模振幅都与各自的无耗散传播参考线 很好地吻合在一起, 说明这两个模态都处在自由传 播的状态; 对于 $(-3,3)$ 模, 无耗散的传播状态可以维 持到 $100 \mathrm{~km}$ 以上. 在过去关于非迁移潮汐诱发机制 的数值模拟研究中, 有关研究人员利用对流活动统 计资料建立了与热带深厚对流活动相关的全球潜热 加热分布, 并将其作为热力驱动源来驱动数值模式 [16,17], 结果指出受这种潜热加热驱动产生的DE3 潮 汐可以无耗散地传播到MLT区域. 事实上, 本文分析 结果指出图 2(a)和(b)中所显示的无耗散传播状态可 以向下延伸到 $20 \mathrm{~km}$ (分析数据所覆盖高度的底部), 与这些模拟研究结果是相当一致的.

在本研究中, 利用能够覆盖直到 $140 \mathrm{~km}$ 的数据集 开展分析的结果对DE3 潮汐活动中心的高度分布取得
了相当详细的认识. 图 2 显示的HMD分析结果清楚地 表明在 $100 \mathrm{~km}$ 以上DE3 活动只受到第一传播模的控 制; 在这里, $(-3,3)$ 模振幅 $\mathrm{N} 3$ 远远大于其他Hough模的 振幅. 与此同时还看到 $\mathrm{N} 3$ 随高度的变化呈现出一个 位于 $110 \mathrm{~km}$ 高度附近的极大值. 基于 $(-3,3)$ 模所表现 出的显著控制作用, 与N 3 极大值出现高度对应, DE3 潮汐活动中心也应该位于 $110 \mathrm{~km}$ 高度. 过去受探测数 据覆盖高度的限制或关注问题角度的不同, 关于这种 潮汐活动的分析结果都没有超过 $95 \mathrm{~km}$ 高度 ${ }^{[1 \sim 4]}$.

与此同时, $110 \mathrm{~km}$ 高度附近 N3 随时间的变化还 能够代表 DE3 潮汐活动中心随时间演变的形式. 图 $3(a)$ 给出了 $111 \mathrm{~km}$ 高度 $(-3,3)$ 模振幅 N3 随时间的演 变. 其中可以看到显著的季节变化, 其特征表现为出 现在北半球夏季的单峰形式，这与过去报告的结果 是一致的(请参考文献[5]以及其中的文献). 除了年变 化形式外, 仔细检查图 3(a)还可以看到, 在分析时段 内 $(-3,3)$ 模振幅 N3 分别在 2002 和 2004 年经历了两 次显著增强的年度峰值, 表现出周期为 $2 \mathrm{a}$ 的变化. 事实上, 当前分析结果揭示在几乎所有中间层和低 热层高度, $(-3,3)$ 模都具有与图 3(a)一致的年变化和 2 
$\mathrm{a}$ 变化, 图 3(b)中给出的 $83 \mathrm{~km}$ 高度 N3 演变过程说 明了这种一致的变化.

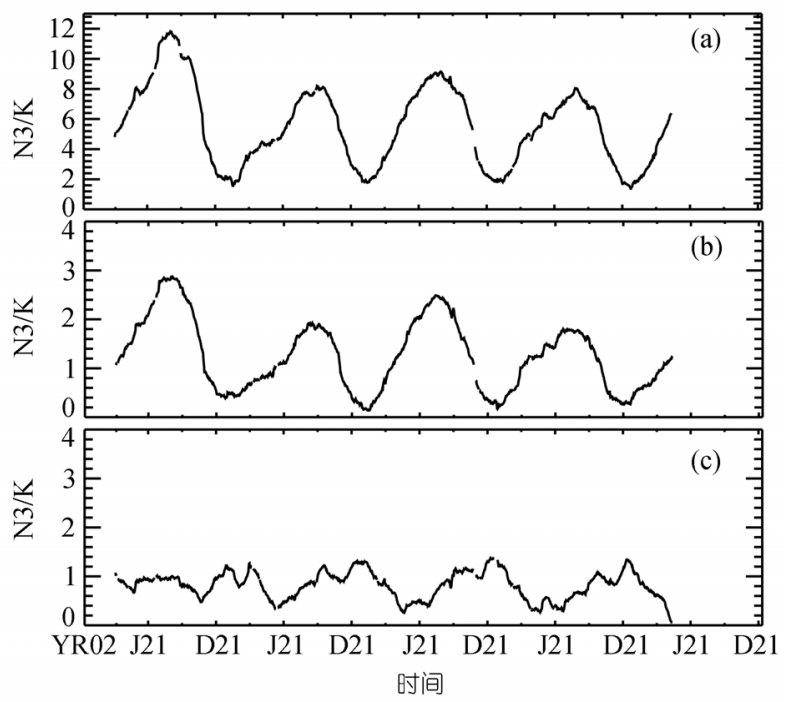

图 3 不同高度、不同 Hough 模振幅(K)距时间的演变

(a) $111 \mathrm{~km},(-3,3)$ 模; (b) $83 \mathrm{~km},(-3,3)$ 模; (c) $83 \mathrm{~km},(-3,4)$ 模. 原 点为 2002 年第一天(YR02); 另外, J 和 D 分别表示 6 和 12 月, 其 后的数字 21 表示日期, 如 J21 表示 06-21

基于上述 HMD 分析结果所揭示的 DE3 活动特 征, 作者对从测量数据中提取的 DE3 潮汐信号进行 了考察, 得到了与上述 HMD 分析完全一致的结果. 图 4(a)首先给出了赤道上 DE3 振幅随高度的时间演 变, 它清楚地说明了 DE3 的活动中心出现在北半球 夏季并且位于 $110 \mathrm{~km}$ 高度的特征. 检查图 4(a)中 N3 极大值中心的演变可以发现除了年变化形式外, 与 图 3(a)中 2 a 周期变化中的峰值对应，赤道上 DE3 潮 汐在 2002 和 2004 年分别出现显著增强的活动, 其中 心振幅达到 $12 \mathrm{~K}$ 以上. 与此同时，作者还考察了 110 $\mathrm{km}$ 高度附近 DE3 振幅的纬度结构及其随时间的变化. 图 4(b)给出了 $107 \mathrm{~km}$ 高度的结果, 其中同样清楚地 显示了 DE3 活动在 2002 和 2004 年显著增强的现象. 通过这些观察说明在 DE3 活动中心高度, 这种潮汐 具有周期为 $2 \mathrm{a}$ 的特征变化.

过去关于 $100 \mathrm{~km}$ 以下DE3 潮汐活动年变化特征 的研究报告都指出这种潮汐主要以反对称于赤道的 形式出现 ${ }^{[1 \sim 5]}$. Forbes和 $\mathrm{Wu}^{[4]}$ 认为这是与 $(-3,4)$ 模在 这些高度存在较强影响有关. 与Forbes和 $\mathrm{Wu}^{[4]}$ 一致, 图 2(b)说明在 $100 \mathrm{~km}$ 以下, $(-3,4)$ 模具有与 $(-3,3)$ 模 相当的振幅，而在 $80 \mathrm{~km}$ 以下它们具有几乎相同的平

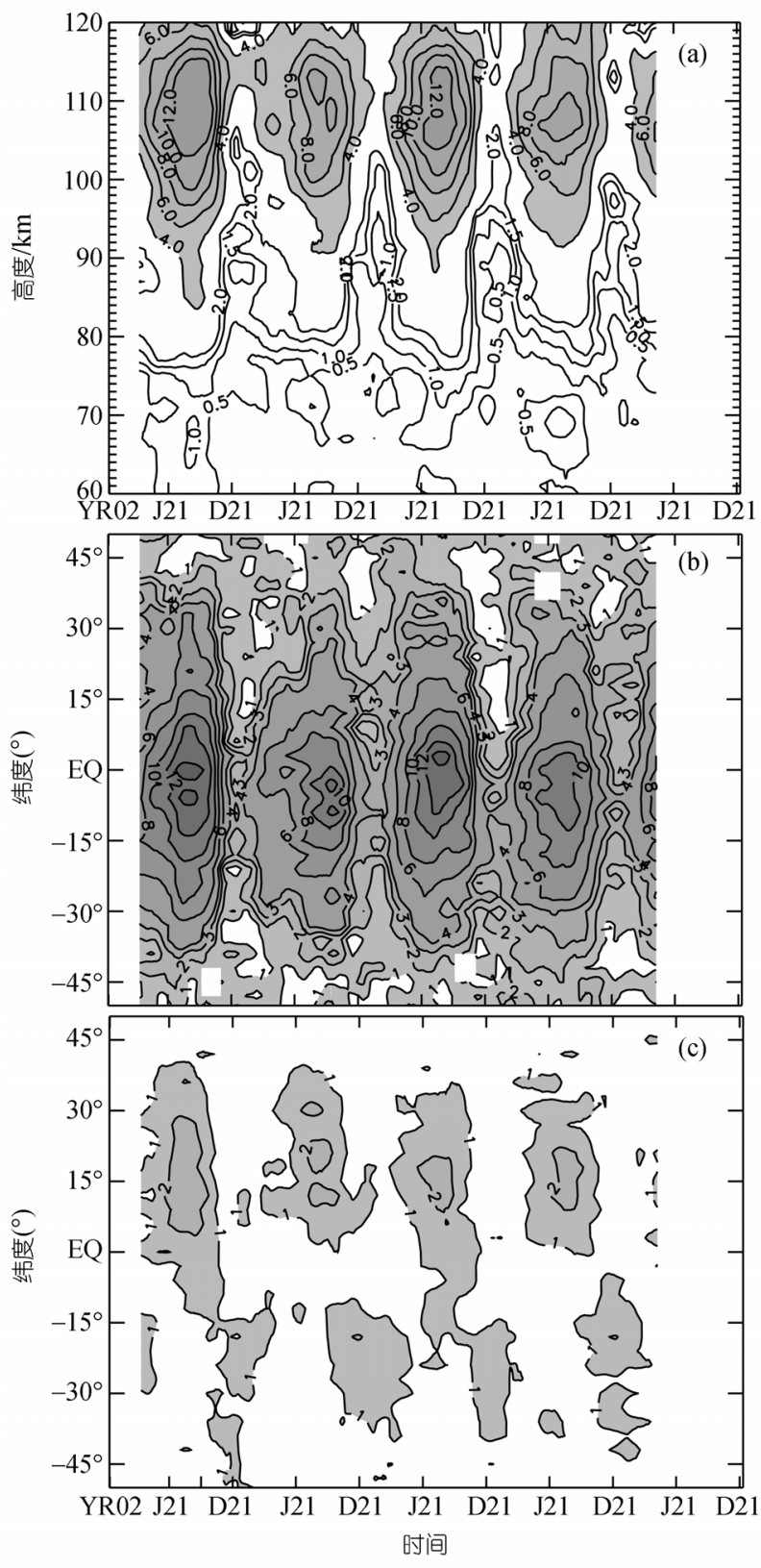

图 4

(a) 赤道上 DE3 振幅(K)在时间-高度剖面中的分布; (b) $107 \mathrm{~km}$, DE3 振幅 $(K)$ 纬度结构随时间演变的形式; (c) $77 \mathrm{~km}, \mathrm{DE} 3$ 振幅 $(\mathrm{K})$ 纬度结构随时间演变的形式

均振幅. 图 3(c)说明在中间层中部存在持续的 $(-3,4)$ 模贡献. 比较图 3(b)和(c)看到, 在 $83 \mathrm{~km}$ 高度, 虽然 $(-3,3)$ 模的峰值显著大于 $(-3,4)$ 模的峰值, 但是它也 表现出显著的年变化幅度; 与此不同, 在所有分析时 段中 $(-3,4)$ 模都持续地维持在一定水平上, 这种来自 $(-3,4)$ 模的持续影响在相当程度上解释了 DE3 所表 
现出的反对称结构.

另外, 比较图 3(b)和(c)还可以看到, 虽然 $(-3,4)$ 模表现出较复杂的演变形式, 但是仍然可以分辨出 双峰年变化的特点. 例如, 图 3(c) 显示 N4 总是在每 年的夏至日附近 (J21)处在极小值状态; 除了这个主 要的极小值外, 在每年冬至日(D21)附近还可以看到 一个较弱的极小值, 这样形成了分别出现在秋末冬 初和冬末春初的两个峰值时段.

进一步观察还发现，随着高度下降，图 3(c)中所 显示的双峰年变化也开始在 DE3 活动中逐渐变得明 显. 图 4(c)给出了 $77 \mathrm{~km}$ 高度 DE3 振幅的纬度结构及 其时间变化. 通过观察发现, 与图 3(b)中 N4 半年变 化位相一致, 中间层下部区域(以 $77 \mathrm{~km}$ 为例)DE3 活 动中心在冬末春初和秋末冬初两个季节交替地出现 在赤道以南和以北. 这些现象说明在这些高度存在 显著地来自 $(-3,4)$ 模的控制作用.

最后值得指出的是, 这两个控制DE3 活动的 Hough模都具有相当深厚的垂直结构. 根据理论计算 结果, 在MLT背景环境中, $(-3,3)$ 模具有约 $56 \mathrm{~km}$ 的 垂直波长, 而 $(-3,4)$ 模具有约 $30 \mathrm{~km}$ 的垂直尺度. 在 本研究中, 通过观察年度峰值时段DE3 潮汐的振幅 和相位的纬度-高度结构得到了与上述理论计算结果 一致的垂直结构(图略). 这样的性质有利于这种潮汐 克服热层底部的耗散而传播到电离层和热层深处. 近来有关研究 ${ }^{[6]}$ 指出电离层中的一些特征扰动具有 与 DE3 一致的纬向结构, 因而推测来自中间层的DE3 传播过程可能是导致这种电离层特征扰动的主要机 制. 因此从考察电离层扰动产生机制角度, 对DE3 的 深入研究需要得到进一步关注.

\section{3 结论}

利用多年卫星探测温度数据提取了中间层和低
热层(MLT)大气热力潮汐信号, 并且利用 Hough 函数 分解(HMD)方案对 DE3 潮汐的全球结构开展考察. HMD 分析结果这种潮汐主要受前两个传播性 Hough 模, 即 $(-3,3)$ 和(-3, 4)模的控制; 这两个 Hough 模都 表现出自由传播的特点, 可以分别自由传播到 100 和 $90 \mathrm{~km}$ 高度. 同时发现, 所有衰减 Hough 模以及其他 传播模的影响是可以忽略的.

根据 HMD 分析结果, 本文给出了 MLT 区域中 DE3 潮汐的整体结构, 指出 DE3 潮汐活动的中心主 要受第一传播 Hough 模, $(-3,3)$ 模的控制, 出现在 110 $\mathrm{km}$ 高度, 表现出稳定的单峰年变化, 最强活动出现 在北半球夏季 (7 月附近). 与此同时, HMD 分析结果 还显示在, 在分析时段内 $(-3,3)$ 模分别在 2002 和 2004 年经历了两次显著增强的年度峰值, 表现出周 期为 $2 \mathrm{a}$ 的变化; 同时还发现, 在几乎所有中间层和 低热层高度, $(-3,3)$ 模都具一致的年变化和 $2 \mathrm{a}$ 变化.

与此同时, 对 DE3 潮汐信号进行考察取得了与 上述 HMD 分析完全一致的结果. 例如, 考察结果表 明 DE3 的活动中心出现在北半球夏季并且位于 110 $\mathrm{km}$ 高度, 它表现出单峰年变化的特征. 在分析时段 内除了年变化形式外, 与 $(-3,3)$ 模 2 a 变化位相一致, DE3 潮汐在 2002 和 2004 年分别出现显著增强的活动, 其中心振幅达到 $12 \mathrm{~K}$ 以上.

分析结果还指出, 在 $100 \mathrm{~km}$ 以下高度, 第二传 播 Hough 模, (-3, 4)模, 也起着重要的影响, 使得这 里的 DE3 潮汐活动表现出关于赤道反对称的结构. 另外, $(-3,4)$ 模表现出双峰年变化的特征, 并且在中 间层下部区域起着显著的控制作用; 例如, 在 $77 \mathrm{~km}$ 高度考察的结果显示, 这里的 DE3 潮汐活动中心出 现在冬末春初和秋末冬初两个季节中, 并且交替地 出现在赤道以南和赤道以北的热带地区.

致谢 本文使用的 SABER/TIMED 数据来自美国 TIMED 计划数据中心(MDC)科学数据系统(SDS)中 SABER 数据发布 网站 http://saber.gats-inc.com. 感谢两位匿名审稿人提出的建设性修改意见.

参考文献

1 Talaat E R, Liberman R S. Nonmigrating diurnal tides in mesospheric and lower thermospheric winds and temperatures. J Atmos Sci, 1999, 56: 4073-4087[DOI]

2 Forbes J, Zhang X, Ward W, et al. Nonmigrating diurnal tides in the thermosphere. J Geophys Res, 2003, 108: 1033, doi: 10.1029/2002JA009262 [DOI] 
3 Manson A H, Meek C, Hagan M, et al. Global distributions of diurnal and semidiurnal tides: Observations from HRDI/UARS of the MLT region and comparisons with gswm-02 (migrating, nonmigrating components). Annu Geophys, 2004, 22: 1529-1548

4 Forbes J, Wu D. Solar tides as revealed by measurements of mesosphere temperature by the MLS experiment on UARS. J Atmos Sci, 2006, 63: 1776-1797[DOI]

5 陈泽宇, 吕达仁. 东经 $120^{\circ} \mathrm{E}$ 中间层和低热层大气潮汐及其季节变化特征. 地球物理学报, 2007, 50(3): 691一 700

6 Wan W, Liu L, Pi X, et al. Wavenumber-4 patterns of the total electron content over the low latitude ionosphere. Geophys Res Lett, 2008, 35: L12104, doi: 10.1029/2008GL033755[DOI]

7 Russell J, Mlynczak M, Gordley L, et al. An overview of the saber experiment and preliminary calibration results. In: Allen M Larar, ed. Proceeding of SPIE. Denver: SPIE, 1999. 3756: 277-288[DOI]

8 Mertens C J, Mlynczak M G, Lopez-Puertas M, et al. Retrieval of mesospheric and lower thermospheric temperature from measurements of $\mathrm{CO}_{2} 15 \mu \mathrm{m}$ Earth limb emission under non-LTE conditions. Geophys Res Lett, 2001, 28(7): 1391-1394[DOI]

9 Mertens C J, Schmidlin F J, Goldberg R A, et al. SABER observations of mesospheric temperatures and comparisons with falling sphere measurements taken during the 2002 summer MaCWAVE campaign. Geophys Res Lett, 2004, 31: L03105, doi: 10.1029/ 2003GL018605[DOD]

10 Siskind D E, Coy L, Espy P. Observations of stratospheric warmings and mesospheric coolings by the TIMED SABER instrument. Geophys Res Lett, 2005, 32: L09804, doi: 10.1029/2005GL022399[DOI]

11 Palo S E, Forbes J M, Zhang X, et al. Planetary wave coupling from the stratosphere to the thermosphere during the 2002 Southern Hemisphere pre-stratwarm period. Geophys Res Lett, 2005, 32: L23809, doi: 10.1029/2005GL024298[DOI]

12 McLandress C, Shepherd G, Solheim B-H. Satellite observations of thermospheric tides: Results from the wind imaging interferometer on UARS. J Geophys Res, 1996, 101(D2): 4093-4114 [DOI]

13 Chapman S, Lindzen R. Atmospheric Tides: Thermal and Gravitational. New York. Gordon and Breach, 1970

14 McLandress C. Seasonal variability of the diurnal tide: Results from the Canadian middle atmosphere general circulation model. J Geophys Res, 1997, 102: 29747-29763[DOI]

15 Flattery T W. Hough Functions. Technical Report. No 21 to NSF. 1967

16 Forbes J, Zhang X, Hagan M E. Simulation of diurnal tides due to tropospheric heating from the NCEP/NCAR reanalysis Project. Geophys Res Lett, 2001, 28: 3851-3854 [DOI]

17 Hagan M E, Forbes J M. Migrating and nonmigrating diurnal tides in the middle and upper atmosphere excited by tropospheric latent heat release. J Geophys Res, 2002, 107: 4754, doi: 10.1029/2001JD001236[DOI] 